\title{
Effects of insulin resistance and hepatic lipid accumulation on hepatic mRNA expression levels of apoB, MTP and L-FABP in non-alcoholic fatty liver disease
}

\author{
NOBITO HIGUCHI ${ }^{1}$, MASAKI KATO ${ }^{1}$, MASATAKE TANAKA ${ }^{1}$, MASAYUKI MIYAZAKI ${ }^{1}$, \\ SHINICHIRO TAKAO ${ }^{1}$, MOTOYUKI KOHJIMA ${ }^{1}$, KAZUHIRO KOTOH ${ }^{1}$, MUNECHIKA ENJOJI $^{2}$, \\ MAKOTO NAKAMUTA ${ }^{3}$ and RYOICHI TAKAYANAGI ${ }^{1}$
}

${ }^{1}$ Department of Medicine and Bioregulatory Science, Graduate School of Medical Sciences, Kyushu University,
Fukuoka 812-8582; ${ }^{2}$ Department of Clinical Pharmacology, Faculty of Pharmaceutical Sciences,
Fukuoka University, Fukuoka $814-0180 ;{ }^{3}$ Department of Gastroenterology, Kyushu Medical Center,
National Hospital Organization, Fukuoka 810-8563, Japan

Received July 11, 2011; Accepted July 25, 2011

DOI: $10.3892 / \mathrm{etm} .2011 .328$

\begin{abstract}
Non-alcoholic fatty liver disease (NAFLD) is considered a hepatic manifestation of metabolic syndrome, which is known to be associated with insulin resistance (IR). NAFLD occurs when the rate of hepatic fatty acid uptake from plasma and de novo fatty acid synthesis is greater than the rate of fatty acid oxidation and excretion as very low-density lipoprotein (VLDL). To estimate the effects of IR on hepatic lipid excretion, mRNA expression levels of genes involved in VLDL assembly were analyzed in NAFLD liver. Twenty-two histologically proven NAFLD patients and 10 healthy control subjects were enrolled in this study. mRNA was extracted from liver biopsy samples and real-time PCR was performed to quantify the expression levels of apolipoprotein B (apoB), microsomal triglyceride transfer protein (MTP) and liver fattyacid binding protein (L-FABP). Hepatic expression levels of the genes were compared between NAFLD patients and control subjects. In NAFLD patients, we also examined correlations between expression levels of the genes and metabolic factors, including IR, and the extent of obesity and hepatic lipid accumulation. Hepatic expression levels of apoB, MTP and L-FABP were significantly up-regulated in NAFLD patients compared to control subjects. The expression levels of MTP were correlated with those of apoB, but not with those of L-FABP. In the NAFLD liver, the expression levels of MTP were significantly reduced in patients with HOMA-IR >2.5. In addition, a signifi-
\end{abstract}

Correspondence to: Dr Masaki Kato, Department of Medicine and Bioregulatory Science, Graduate School of Medical Sciences, Kyushu University, 3-1-1 Maidashi, Higashi-ku, Fukuoka 812-8582, Japan E-mail: mkato11@intmed3.med.kyushu-u.ac.jp

Key words: apolipoprotein B, fatty-acid binding protein, homeostasis model assessment of insulin resistance, microsomal triglyceride transfer protein, non-alcoholic fatty liver disease, very low-density lipoprotein cant reduction in MTP expression was observed in livers with advanced steatosis. Enhanced expression of genes involved in VLDL assembly may be promoted to release excess lipid from NAFLD livers. However, the progression of IR and hepatic steatosis may attenuate this compensatory process.

\section{Introduction}

Non-alcoholic fatty liver disease (NAFLD) is a clinicopathological disease characterized by elevated accumulation of triglycerides in the liver. In more than $10 \%$ of patients with NAFLD, liver disease progresses to non-alcoholic steatohepatitis (NASH), which is characterized by hepatic inflammatory cell infiltration and ballooning of hepatocytes. Liver cirrhosis and hepatocellular carcinoma occur in certain patients with NASH (1).

Many studies have attempted to investigate the mechanisms involved in the progression of hepatic steatosis to control lipid content in NAFLD. Steatosis occurs when the rate of hepatic fatty acid uptake from plasma and de novo fatty acid synthesis is greater than the rate of fatty acid oxidation and excretion as very low-density lipoprotein (VLDL) (2). Insulin resistance (IR), which is frequently accompanied by obesity and T2DM, is known to promote the progression of hepatic steatosis (3). Of note, insulin activity to suppress triglyceride hydrolysis in adipose tissue is diminished in IR, eventually causing fatty acid accumulation in the liver (4). Moreover, enhanced hepatic lipid production is induced by IR. Hyperglycemia induces the transactivation of transcriptional factors, carbohydrate responsive element binding protein and Liver $\mathrm{X}$ receptor, which are known to activate de novo hepatic lipid synthesis $(5,6)$. Furthermore, triglyceride hydrolysis in the liver is also modified by IR. Hepatic mRNA expression levels of lipolytic enzymes, hormone-sensitive lipase and adipose tissue triglyceride lipase were found to be suppressed in NAFLD with IR (7). These observations suggest that IR may adversely affect multiple mechanisms regulating hepatic lipid contents and promote the progression of NAFLD. 
Triglyceride release as VLDL requires coordinated functions of apolipoprotein $\mathrm{B}(\mathrm{apoB})$ and microsomal triglyceride transfer protein (MTP) (8). ApoB is an essential protein required for assembly and secretion of VLDL from the liver and chylomicron from the small intestine (9). MTP transfers free cholesterol, phospholipids, triglyceride and cholesterol esters to apoB during translation, allowing apoB to attain a preVLDL conformation, which supports the subsequent fusion of apoB with MTP-stabilized triglyceride droplets and the formation of mature VLDL (10). Genetic analyses confirm the crucial roles of MTP in lipid metabolism; liver-specific Mttp-knockout induced striking reductions in VLDL triglyceride and caused hepatic steatosis (11). In addition, liver fatty-acid binding protein (L-FABP) is known to facilitate intracellular trafficking of long-chain fatty acids (LCFAs) (12). This property of L-FABP modulates diverse cellular functions, including fatty acid uptake and intracellular lipid contents and metabolism.

To understand the effects of IR on the mechanism of triglyceride release from the liver, we conducted this study to estimate hepatic mRNA expression levels of apoB, MTP and L-FABP in patients with NAFLD using RT-PCR. We examined the effects of metabolic factors, including IR, and the extent of obesity and hepatic lipid accumulation on the expression of the genes. We found that the expression levels of all genes examined in the NAFLD liver were significantly increased compared to those of the healthy controls. Furthermore, IR or advanced hepatic steatosis significantly reduced the expression levels of MTP in the NAFLD liver. These observations may be helpful to understand the effects of IR on the progression of NAFLD.

\section{Materials and methods}

Patients and samples. Liver tissue samples were obtained by biopsy from 22 patients with histologically diagnosed NAFLD who were admitted to the Kyushu University Hospital between 2004 and 2006. To avoid the effects of fibrosis on metabolic parameters, patients histologically diagnosed with NASH were not included. Liver tissue samples were obtained from living donors during liver transplantation and used as healthy controls. Written consent was obtained from all of the patients. Characteristics of the enrolled subjects, including gender, age, body mass index (BMI), serum levels of aspartate aminotransferase (AST), alanine aminotransferase (ALT), $\gamma$-glutamyl transpeptidase $(\gamma \mathrm{GTP})$, lactate dehydrogenase $(\mathrm{LDH})$, total cholesterol, triglyceride, fasting plasma glucose, C-reactive protein $(\mathrm{CRP})$, platelet count and prothrombin time, were documented.

$R T-P C R$. Total RNA was prepared with TRIzol reagent (Invitrogen, Carlsbad, CA, USA), and cDNA was synthesized with GeneAmp ${ }^{\text {TM }}$ RNA PCR (Applied Biosystems. Branchburg, NJ, USA). Real-time PCR was performed using LightCycler-FastStart DNA Master SYBR Green 1 (Roche, Basel, Switzerland) according to the manufacturer's instructions. To control for variations in the reactions, all PCR data were normalized against $\beta$-actin expression. PCR primers for apoB were forward 5'-GCCGAGTTTGCCTTGCTCA-3' and reverse 5'-TCCGGAGGCTCACCAGTTTC-3'; the primers for MTP were forward 5'-GGCTCCTGCCTACAGCTTCT-3' and reverse 5'-CAGCCAGTGGATCACCACA-3'; the primers for
Table I. Clinical characteristics of the patients with NAFLD and control subjects.

\begin{tabular}{lccc}
\hline & $\begin{array}{c}\text { Control } \\
(\mathrm{n}=10)\end{array}$ & $\begin{array}{c}\text { NAFLD } \\
(\mathrm{n}=22)\end{array}$ & p-value \\
\hline Male/female & $6 / 4$ & $12 / 10$ & 0.77000 \\
Age (years) & $32.6 \pm 7.06$ & $38.0 \pm 14.1$ & 0.06600 \\
BMI (kg/m $\left.{ }^{2}\right)$ & $21.3 \pm 2.58$ & $25.3 \pm 6.94$ & $0.00261^{\text {a }}$ \\
AST (U/l) & $15.0 \pm 3.35$ & $39.9 \pm 47.1$ & 0.15100 \\
ALT (U/l) & $12.3 \pm 4.09$ & $54.2 \pm 69.6$ & 0.10300 \\
$\gamma$ GTP (U/l) & $17.9 \pm 3.18$ & $61.3 \pm 82.0$ & 0.12900 \\
LDH (U/l) & $138 \pm 27.6$ & $217 \pm 83.2$ & $0.01450^{\text {a }}$ \\
Total cholesterol (mg/dl) & $164 \pm 26.7$ & $191 \pm 45.1$ & 0.12600 \\
Triglyceride (mg/dl) & $77.0 \pm 22.0$ & $130 \pm 112$ & 0.20200 \\
Fasting plasma & $87.6 \pm 4.90$ & $108 \pm 32.2$ & 0.09690 \\
glucose (mg/dl) & & & \\
Platelets (x10 $\left./ \mathrm{mm}{ }^{3}\right)$ & $24.2 \pm 4.39$ & $21.6 \pm 3.77$ & 0.13600 \\
Prothrombin time $(\%)$ & $88.8 \pm 6.65$ & $94.3 \pm 9.32$ & 0.13700 \\
CRP (mg/dl) & $0.062 \pm 0.13$ & $0.081 \pm 0.1$ & 0.69000 \\
\hline
\end{tabular}

BMI, body mass index; AST, aspartate aminotransferase; ALT, alanine aminotransferase; $\gamma \mathrm{GTP}, \gamma$-glutamyl transpeptidase; LDH, lactate dehydrogenase; CRP, C-reactive protein. p-values were calculated to compare the subjects in the control and NAFLD groups $\left({ }^{\mathrm{a}} \mathrm{p}<0.05\right)$.

\section{L-FABP were forward 5'-CAGCTGCGGGATGAGATTGA-3' and reverse 5'-AAACGCTGGTGTGTGATGGGTA-3'.}

Statistical analyses. The results were expressed as the means \pm standard deviation. Significant differences between two groups were assessed using unpaired two-tailed t-tests. A value of $\mathrm{p}<0.05$ was considered significant.

\section{Results}

Subject characteristics. We studied 22 NAFLD patients (12 males and 10 females) and 10 control subjects (6 males and 4 females) (Table I). Since control samples were obtained from healthy donors for transplantation, the age of this group was younger compared to the NAFLD group, but the difference was not significant. BMI was significantly higher in the NAFLD group than in the control group. AST, ALT and $\gamma$ GTP were higher in the NAFLD group than in the control group, but the differences were not significant. Nutritional parameters, including total cholesterol, triglyceride and fasting plasma glucose, were higher in the NAFLD group than in the control group, but the differences were not statistically significant.

Up-regulation of genes involved in VLDL assembly and fatty acid trafficking in the NAFLD liver. mRNA expression levels of apoB and MTP were significantly higher, 3-fold and 2-fold, respectively, in the NAFLD than in the control liver (Fig. 1A and B). L-FABP was also significantly higher in the NAFLD liver than in the control (Fig. 1C). Since all three genes were up-regulated in the NAFLD liver in a similar manner, we evaluated the correlations among the expression levels of these genes. As shown in Fig. 2, the expression levels of MTP 
A

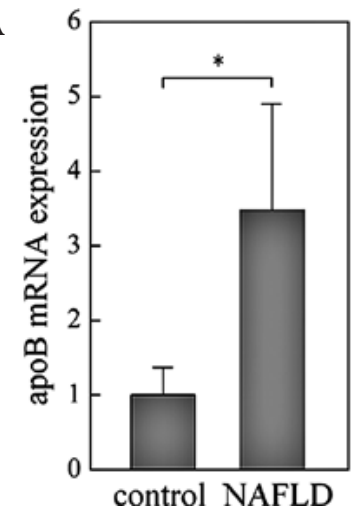

B

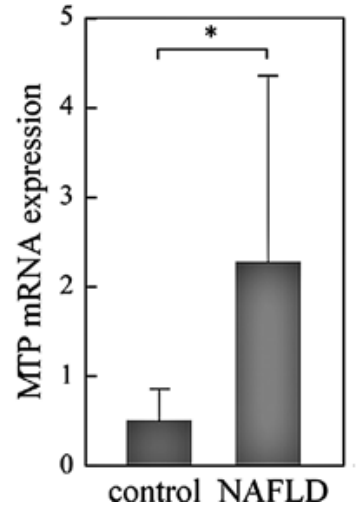

C

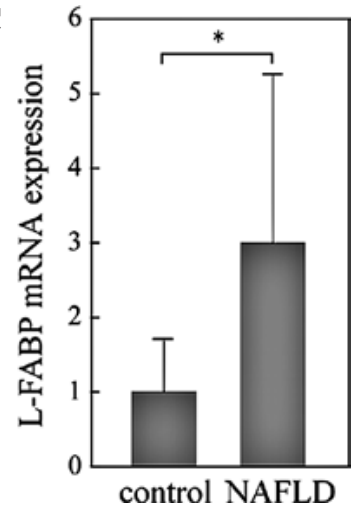

Figure 1. RT-PCR analyses of the expression levels of genes involved in VLDL assembly and fatty acid trafficking in NAFLD and control livers. Total RNA was extracted from liver biopsy samples of NAFLD patients $(n=22)$ and control subjects $(n=10)$. Samples were analyzed by RT-PCR and normalized for $\beta$-actin expression. (A) apoB; (B) MTP; (C) L-FABP. The mean expression in the control liver was set as $1 .{ }^{*} \mathrm{p}<0.05$.

A

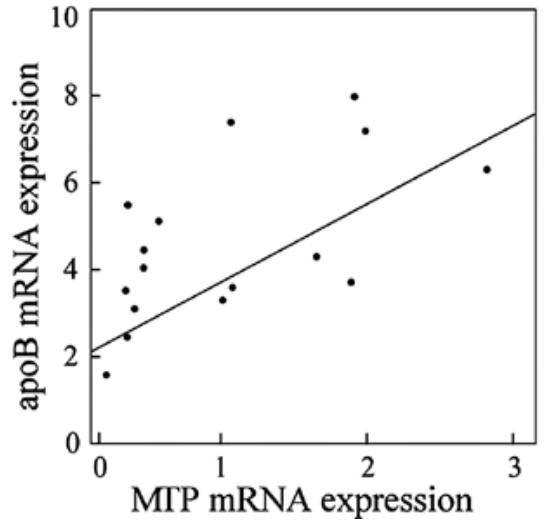

B

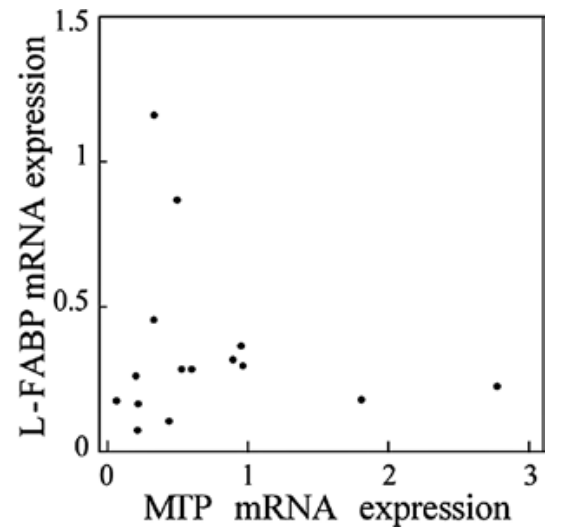

Figure 2. Correlations between the expression levels of genes involved in VLDL assembly and fatty acid trafficking in NAFLD liver. (A) apoB and MTP; (B) L-FABP and MTP. Expression levels of MTP were significantly correlated with those of apoB ( $\mathrm{r}=0.614$, $\mathrm{p}=0.0006$ ), but not with those of L-FABP.

were significantly correlated with those of apoB $(\mathrm{r}=0.614$, $\mathrm{p}=0.0006$; Fig. 2A), but not with those of L-FABP (Fig. 2B), suggesting that a common mechanism may be involved in the transcriptional regulation of MTP and apoB in the NAFLD liver. Moreover, enhanced expression of these genes suggests that a compensatory mechanism to release excess lipids is activated in the NAFLD liver.

Effects of IR on the expression levels of apoB, MTP and $L-F A B P$ in the NAFLD liver. We analyzed whether IR modifies the expression levels of these genes in NAFLD. In 22 patients with NAFLD, 12 had homeostasis model assessment of insulin resistance (HOMA-IR) values $>2.5$ and were considered to have IR. Expression levels of all of the genes measured were reduced in patients with IR. Of note, the expression levels of MTP in patients with HOMA-IR > 2.5 were significantly reduced compared to those with HOMA-IR $<2.5$ (Fig. 3). To evaluate the effects of obesity, we compared the expression levels of genes between patients with BMI $<30 \mathrm{~kg} / \mathrm{m}^{2}(\mathrm{n}=9)$ and those with BMI $>30 \mathrm{~kg} / \mathrm{m}^{2}(\mathrm{n}=13)$. However, significant differences were not observed (Fig. 4).

Effects of the extent of lipid accumulation on the expression levels of apoB, MTP and L-FABP in the NAFLD liver. The numbers of lipid-laden hepatocytes in the NAFLD liver were histologically counted on biopsy sections. mRNA expression levels of genes were then compared between liver samples with mild steatosis $(<30 \%$ of hepatocytes showed marked lipid accumulation; $n=12)$ and advanced steatosis $(>30 \%$ of hepatocytes showed marked lipid accumulation; $n=10)$. The expression levels of MTP in livers with advanced steatosis were significantly reduced compared to those with mild steatosis (Fig. 5). A similar tendency was observed in the expression levels of apoB, but the difference was not significant. These results suggest that the reduced expression of MTP in the liver with advanced steatosis is involved in the mechanism to exacerbate hepatic lipid accumulation in the NAFLD liver, through the reduction of lipid excretion as VLDL.

\section{Discussion}

In this study, the hepatic mRNA expression levels of apoB, MTP and L-FABP were increased in the NAFLD liver compared to those in the control livers, suggesting that a compensatory mechanism to release excess lipid as VLDL is promoted in the steatotic liver. However, in patients with IR or advanced steatosis, this compensatory mechanism seems to be disrupted via the down-regulation of MTP. This process to attenuate the compensatory mechanism may be involved in the progression of NAFLD. 

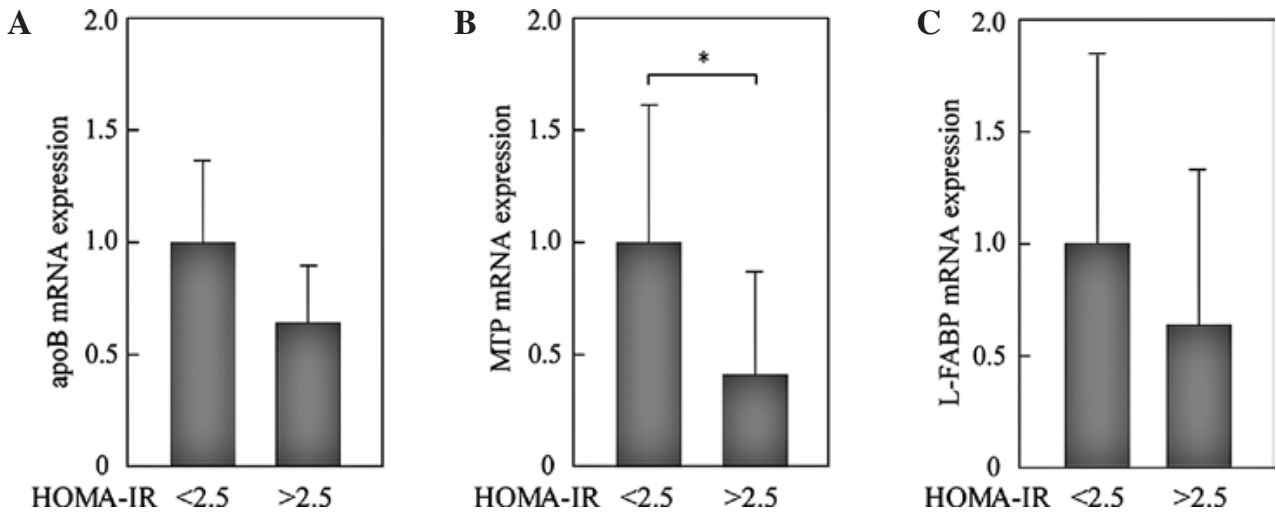

Figure 3. Effects of IR on the expression of genes involved in VLDL assembly and fatty acid trafficking in NAFLD liver. Patients with HOMA-IR $>2.5$ were considered to have IR. The expression levels of genes were compared between patients with HOMA-IR $<2.5(\mathrm{n}=10)$ and those with HOMA-IR $>2.5$ ( $\mathrm{n}=12)$. All PCR reactions were normalized for $\beta$-actin expression. (A) apoB; (B) MTP; (C) L-FABP. The mean expression in the patients with HOMA-IR <2.5 was set as $1 .{ }^{*} \mathrm{p}<0.05$.
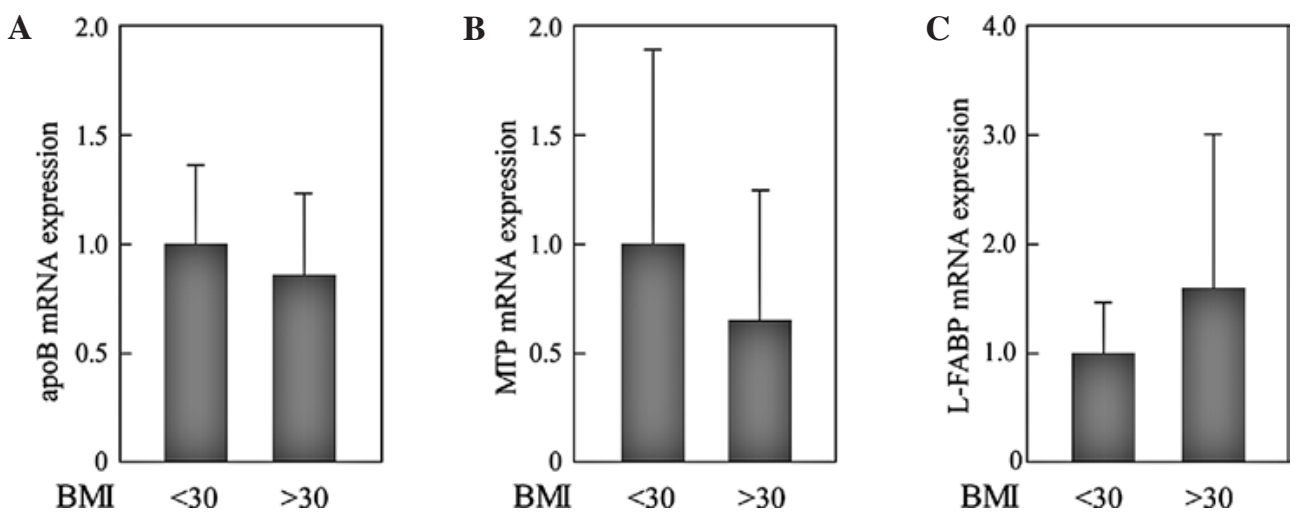

Figure 4. Effects of BMI on the expression of genes involved in VLDL assembly and fatty acid trafficking in NAFLD liver. The expression levels of genes were compared between patients with BMI $<30 \mathrm{~kg} / \mathrm{m}^{2}(\mathrm{n}=9)$ and those with BMI $>30 \mathrm{~kg} / \mathrm{m}^{2}(\mathrm{n}=13)$. All PCR reactions were normalized for $\beta$-actin expression (A) apoB; (B) MTP; (C) L-FABP. The mean expression in the patients with BMI $<30 \mathrm{~kg} / \mathrm{m}^{2}$ was set as 1 . " $\mathrm{p}<0.05$.
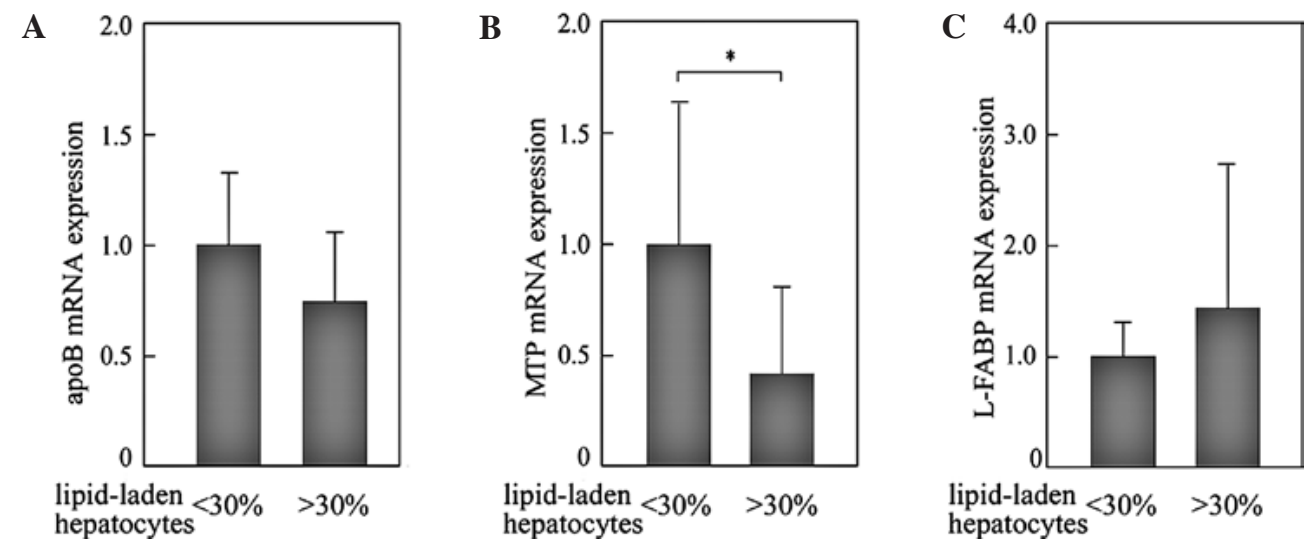

Figure 5. Effects of the extent of lipid accumulation on the expression of genes in NAFLD liver. The numbers of hepatocytes with marked lipid accumulation were counted on biopsy sections. The expression levels of genes were compared between the liver samples with mild steatosis (<30\% of hepatocytes show marked lipid accumulation; $\mathrm{n}=12)$ and in liver samples with advanced steatosis $(>30 \%$ of hepatocytes show marked lipid accumulation; $\mathrm{n}=10)$. All PCR reactions were normalized for $\beta$-actin expression. The mean expression in NAFLD liver with mild steatosis was set as $1 .{ }^{*} \mathrm{p}<0.05$.

Transcriptional regulations of genes examined in this study are known to be mediated by transcriptional factors, including hepatocyte nuclear factor (HNF) $4 \alpha$ and peroxisome proliferator-activated receptor (PPAR) $\alpha$. HNF4 $\alpha$ controls the expression of genes involved in lipoprotein and lipid metabolism, including MTP and apoB (13-15). HNF4 $\alpha$ binds long chain fatty acyl-CoA and this binding stimulates its transcriptional activity $(16,17)$. PPAR $\alpha$, which regulates the expression of a number of genes critical for lipid and lipoprotein metabolism, is known to transactivate MTP expression in rat liver and cultured hepatocytes $(18,19)$. PPAR $\alpha$ is also responsible for the induction of L-FABP in hepatocytes in response to HMG-CoA 
reductase inhibitor (20). Since our observations showed a positive correlation between the expression levels of apoB and MTP, but not between those of L-FABP and MTP, HNF4 $\alpha$ may be more likely to be responsible for the simultaneous and correlated transactivation of apoB and MTP.

In this study, the expression levels of MTP were reduced in NAFLD with IR; however, this observation is not consistent with the known effects of insulin on the transcriptional regulation of MTP. Hepatic expression of MTP is reported to be down-regulated by insulin (21). In detail, MTP expression is induced by transcriptional factor forkhead box $\mathrm{O} 1$ (FoxO1), whose activation is down-regulated by exposure to insulin $(10,22)$. In response to insulin, FoxO1 is phosphorylated by activated Akt, resulting in a reduction in nuclear FoxO1 and time-dependent decreases in MTP transcription. These findings suggest that MTP expression would not be downregulated in IR. Currently, we cannot explain why the opposite results were observed in this study. Small sample size may be the reason for this unexpected MTP expression. However, we hypothesize that deteriorated cellular circumstances in the NAFLD liver may be responsible. Of note, oxidative stress, which is induced by mitochondrial dysfunction in steatotic cells, has a strong potential to damage various cellular functions, including homeostasis of endoplasmic reaction, control of cell death and transcriptional regulation of various genes (23-26). Furthermore, hepatic iron load in the NAFLD liver would be a factor to enhance cellular damages. Since iron has a strong potential to produce oxidative stress, hepatic iron load is known to promote the progression of NASH $(27,28)$. In the NAFLD liver, reduction in MTP expression levels and increased iron score were simultaneously observed during the progression of liver disease (29). Taken together, reduced MTP expression in NAFLD liver with IR or advanced steatosis may be induced by enhanced oxidative stress in these cellular circumstances.

In this study, the expression of genes involved in VLDL assembly and fatty acid trafficking was enhanced in the liver with NAFLD. However, the reduced expression of MTP in livers with IR or advanced steatosis may be factors to accelerate further steatosis. Further studies are necessary to determine the effects of IR on the progression of NAFLD.

\section{References}

1. Angulo P: Nonalcoholic fatty liver disease. N Engl J Med 346 : 1221-1231, 2002.

2. Fabbrini E, Sullivan S and Klein S: Obesity and nonalcoholic fatty liver disease: biochemical, metabolic, and clinical implications. Hepatology 51: 679-689, 2010.

3. Tiniakos DG, Vos MB and Brunt EM: Nonalcoholic fatty liver disease: pathology and pathogenesis. Annu Rev Pathol 5: 145-171, 2010.

4. Jaworski K, Sarkadi-Nagy E, Duncan RE, Ahmadian M and Sul HS: Regulation of triglyceride metabolism. IV. Hormonal regulation of lipolysis in adipose tissue. Am J Physiol Gastrointest Liver Physiol 293: G1-G4, 2007.

5. Iizuka K and Horikawa Y: ChREBP: a glucose-activated transcription factor involved in the development of metabolic syndrome. Endocr J 55: 617-624, 2008.

6. Higuchi N, Kato M, Shundo Y, Tajiri H, Tanaka M, Yamashita N, Kohjima M, Kotoh K, Nakamuta M, Takayanagi R and Enjoji M: Liver X receptor in cooperation with SREBP-1c is a major lipid synthesis regulator in nonalcoholic fatty liver disease. Hepatol Res 38: 1122-1129, 2008
7. Kato M, Higuchi N and Enjoji M: Reduced hepatic expression of adipose tissue triglyceride lipase and CGI-58 may contribute to the development of non-alcoholic fatty liver disease in patients with insulin resistance. Scand J Gastroenterol 43: 1018-1019, 2008

8. Meshkani R and Adeli K: Hepatic insulin resistance, metabolic syndrome and cardiovascular disease. Clin Biochem 42: 1331-1346, 2009.

9. Shelness GS and Sellers JA: Very-low-density lipoprotein assembly and secretion. Curr Opin Lipidol 12: 151-157, 2001.

10. Sparks JD and Sparks CE: Overindulgence and metabolic syndrome: is FoxO1 a missing link? J Clin Invest 118: 2012-2015, 2008.

11. Raabe M, Veniant MM, Sullivan MA, Zlot CH, Bjorkegren J, Nielsen LB, Wong JS, Hamilton RL and Young SG: Analysis of the role of microsomal triglyceride transfer protein in the liver of tissue-specific knockout mice. J Clin Invest 103: 1287-1298, 1999.

12. Binas B and Erol E: FABPs as determinants of myocellular and hepatic fuel metabolism. Mol Cell Biochem 299: 75-84, 2007.

13. Sladek FM: Orphan receptor HNF-4 and liver-specific gene expression. Receptor 3: 223-232, 1993.

14. Sheena V, Hertz R, Nousbeck J, Berman I, Magenheim J and Bar-Tana J: Transcriptional regulation of human microsomal triglyceride transfer protein by hepatocyte nuclear factor-4alpha. J Lipid Res 46: 328-341, 2005.

15. Ladias JA, Hadzopoulou-Cladaras M, Kardassis D, Cardot P, Cheng J, Zannis V and Cladaras C: Transcriptional regulation of human apolipoprotein genes ApoB, ApoCIII, and ApoAII by members of the steroid hormone receptor superfamily HNF-4, ARP-1, EAR-2, and EAR-3. J Biol Chem 267: 15849-15860, 1992.

16. Hertz R, Magenheim J, Berman I and Bar-Tana J: Fatty acyl-CoA thioesters are ligands of hepatic nuclear factor-4alpha. Nature 392: 512-516, 1998.

17. Jump DB: Dietary polyunsaturated fatty acids and regulation of gene transcription. Curr Opin Lipidol 13: 155-164, 2002.

18. Yoon M: The role of PPARalpha in lipid metabolism and obesity: focusing on the effects of estrogen on PPARalpha actions. Pharmacol Res 60: 151-159, 2009.

19. Ameen C, Edvardsson U, Ljungberg A, Asp L, Akerblad P, Tuneld A, Olofsson SO, Linden D and Oscarsson J: Activation of peroxisome proliferator-activated receptor alpha increases the expression and activity of microsomal triglyceride transfer protein in the liver. J Biol Chem 280: 1224-1229, 2005.

20. Landrier JF, Thomas C, Grober J, Duez H, Percevault F, Souidi M, Linard C, Staels B and Besnard P: Statin induction of liver fatty acid-binding protein (L-FABP) gene expression is peroxisome proliferator-activated receptor-alpha-dependent. J Biol Chem 279: 45512-45518, 2004.

21. Au WS, Kung HF and Lin MC: Regulation of microsomal triglyceride transfer protein gene by insulin in HepG2 cells: roles of MAPKerk and MAPKp38. Diabetes 52: 1073-1080, 2003.

22. Kamagate A, Qu S, Perdomo G, Su D, Kim DH, Slusher S, Meseck $\mathrm{M}$ and Dong HH: FoxO1 mediates insulin-dependent regulation of hepatic VLDL production in mice. J Clin Invest 118: 2347-2364, 2008

23. Wei Y, Wang D, Topczewski F and Pagliassotti MJ: Saturated fatty acids induce endoplasmic reticulum stress and apoptosis independently of ceramide in liver cells. Am J Physiol Endocrinol Metab 291: E275-E281, 2006.

24. Roebuck KA: Oxidant stress regulation of IL-8 and ICAM-1 gene expression: differential activation and binding of the transcription factors AP-1 and NF-kB (Review). Int J Mol Med 4: 223-230, 1999

25. Gius D, Botero A, Shah S and Curry HA: Intracellular oxidation/ reduction status in the regulation of transcription factors NF-kappaB and AP-1. Toxicol Lett 106: 93-106, 1999.

26. Liu H, Colavitti R, Rovira II and Finkel T: Redox-dependent transcriptional regulation. Circ Res 97: 967-974, 2005.

27. George DK, Goldwurm S, MacDonald GA, Cowley LL, Walker NI, Ward PJ, Jazwinska EC and Powell LW: Increased hepatic iron concentration in nonalcoholic steatohepatitis is associated with increased fibrosis. Gastroenterology 114: 311-318, 1998.

28. Mantena SK, King AL, Andringa KK, Eccleston HB and Bailey SM: Mitochondrial dysfunction and oxidative stress in the pathogenesis of alcohol- and obesity-induced fatty liver diseases. Free Radic Biol Med 44: 1259-1272, 2008.

29. Mitsuyoshi H, Yasui K, Harano Y, Endo M, Tsuji K, Minami M, Itoh Y, Okanoue T and Yoshikawa T: Analysis of hepatic genes involved in the metabolism of fatty acids and iron in nonalcoholic fatty liver disease. Hepatol Res 39: 366-373, 2009. 